\title{
Postscript on the indexing of the journal New Physics: Sae Mulli in Scopus
}

\section{Essay}

\author{
Sang-Don Bu', Guinyun Kim², Chang-Hwan Lee ${ }^{3}$, Sungmin $\mathrm{Im}^{4}$
}

'Department of Physics Chonbuk National University, Jeonju; ${ }^{2}$ Department of Physics Kyungpook National University, Gyeongsan; ${ }^{3}$ Department of Physics Pusan National University, Busan; ${ }^{4}$ Department of Physics Education Daegu University, Gyeongsan, Korea

\section{Introduction}

The Korean Physical Society has worked hard to get its journal New Physics: Sae Mulli indexed in Scopus. First published in 1961, the journal has gone through repeated changes and constant development. Such changes include the merging with the journal of Physics Education: Mulli Kyoyuk in 1999, the switch to monthly issues in 2000, the revision of its English name in 2010, the continuous effort of the Korean Physical Society's various regional branches, the committed effort of the editorial board, the creation in 2013 of the English homepage for New Physics: Sae Mulli (http://www.npsm-kps.org), the recruitment of foreign editors, and the introduction of a cover and highlight papers.

Finally, on August 22, 2015, a representative from Elsevier notified us that New Physics: Sae Mulli had been indexed in Scopus, the world's largest citation index database. At present, with over 180 academic and review papers published in twelve monthly issues on the last day of every month, our journal is contributing to the development of the physics community in South Korea.

\section{The Oldest Journal of Korean Physical Society}

Received: January 29, 2016 Accepted: February 3, 2016

Correspondence to Sang-Don Bu sbu@jbnu.ac.kr

ORCID

Sang-Don Bu

http://orcid.org/0000-0003-4883-1308

Guinyun Kim

http://orcid.org/0000-0002-3482-9082

Chang-Hwan Lee

http://orcid.org/0000-0003-3221-1171

Sungmin Im

http://orcid.org/0000-0001-6547-7027
First published in May of 1961, New Physics: Sae Mulli (ISO abbreviation, New Phys.: Sae Mulli) has been the voice of the Korean Physical Society for half a century. In the future as well, the journal should serve as the door through which the Korean Physical Society enters the global stage. One of the oldest journals in Korean science, it has continuously developed over the past 54 years, despite the limitations of being a Korean-language journal.

Let's examine some of the reasons for New Physics: Sae Mullis steady development. First, after its publication, New Physics was successful at drawing in a broad scope of readers. As the articles were written in Korean, they were easy for people to read and were disseminated widely. Physicists, scientists and engineers doing work related to physics, and physics professors as well as students became regular readers of New Physics: Sae Mulli. For these reasons, the journal has been the driving force for the Korean Physical Society's development, and that tradition 


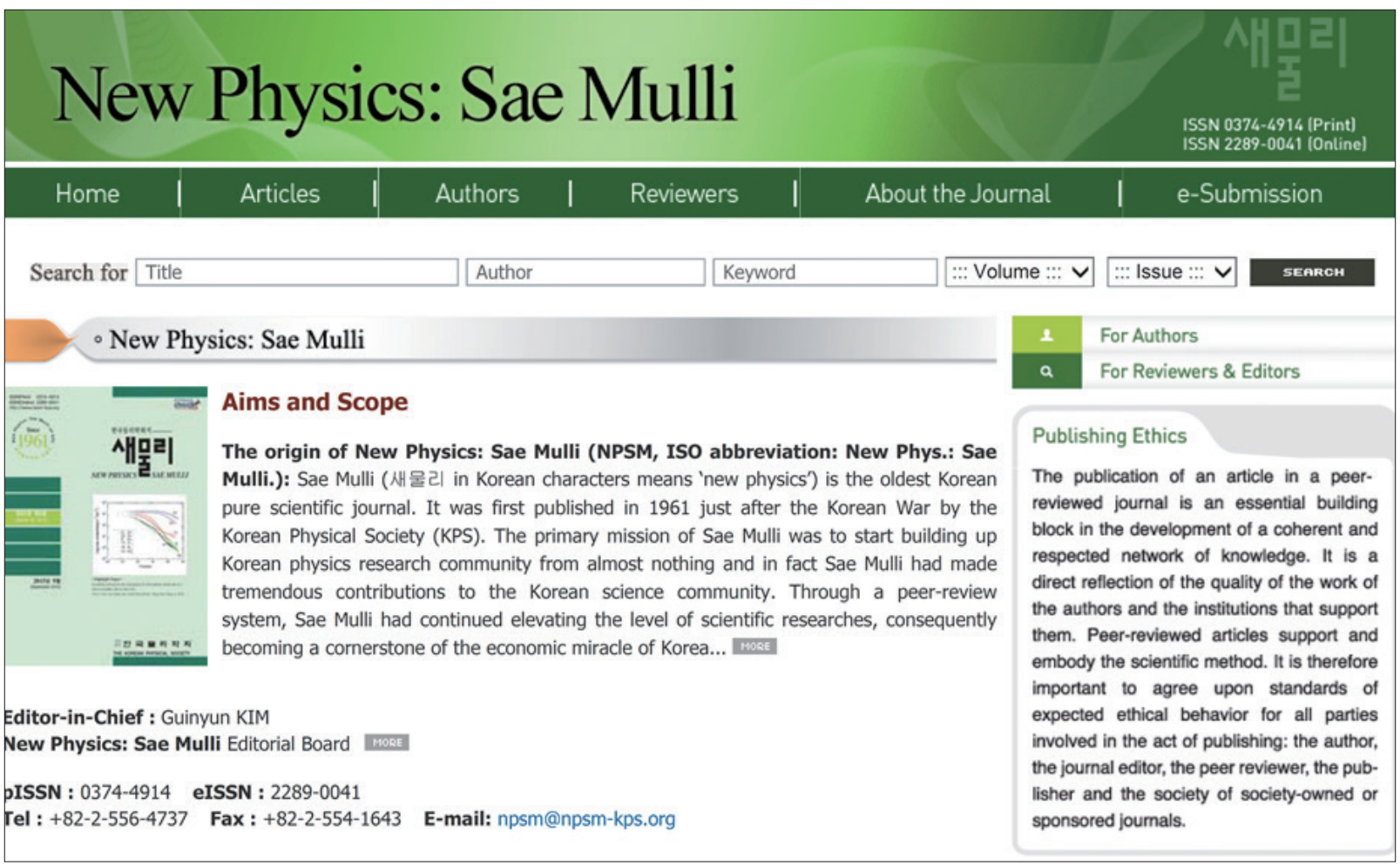

Fig. 1. New Physics:Sae Mulli homepage (http://www.npsm-kps.org).
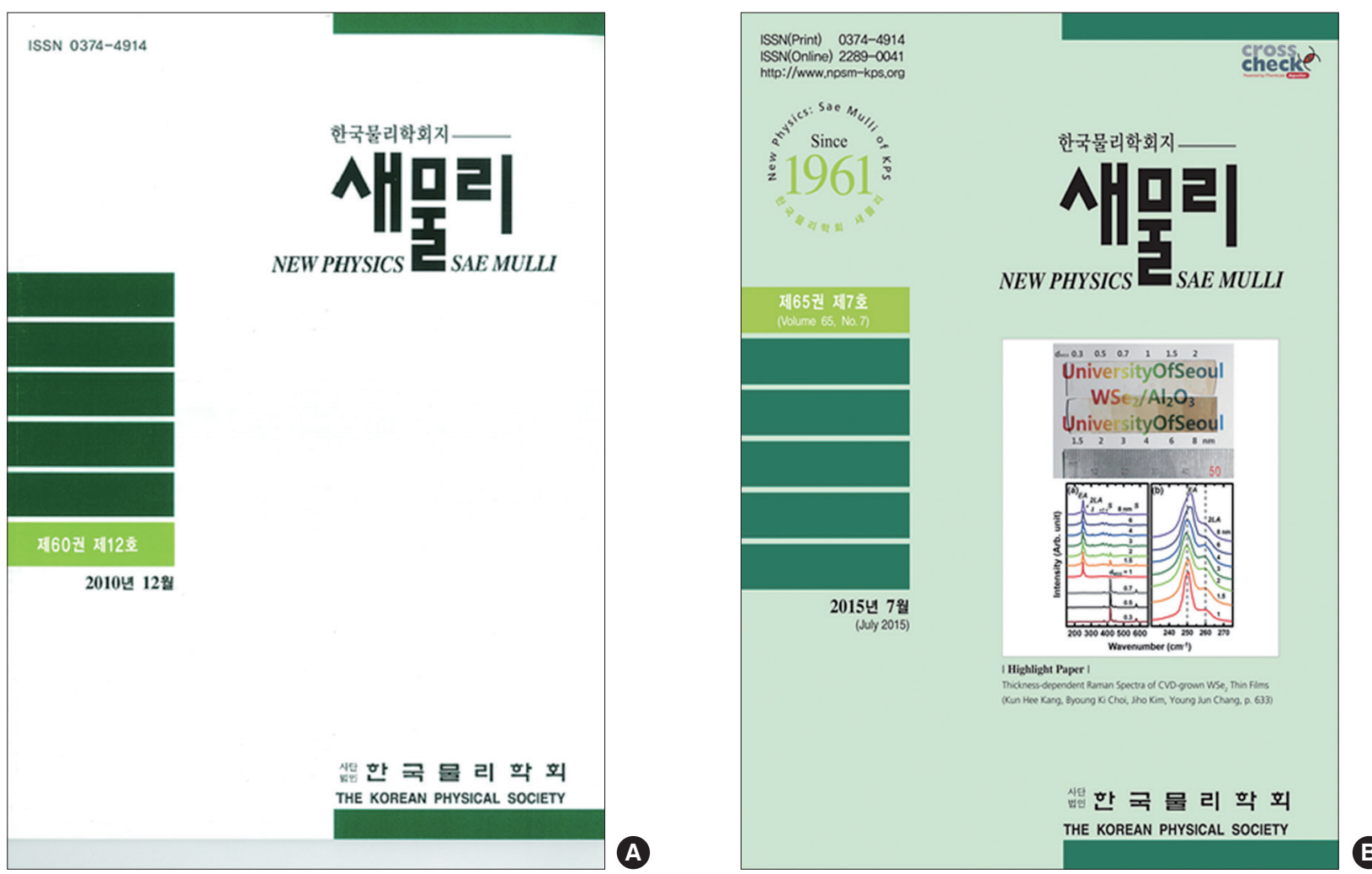

Fig. 2. Improvements in the cover page of New Physics: Sae Mulli. (A) 2010 December issue and (B) 2015 July issue. 
continues up to this day.

Such a broad readership is the primary advantage that New Physics has when compared with other English-medium journals. Second, in order to inform other countries about the results of Korean physics research, New Physics does not merely publish Korean language articles, but has, even since its first issue, included abstracts written in English. For reasons such as these, New Physics was eligible to be indexed in Physics Abstracts (now INSPEC) from the time of its inception in 1961.

\section{Recent Efforts toward International Journal}

In 2010, New Physics went beyond publishing only articles written in Korean and began publishing English-language articles as well, and taking into consideration its international audience, its title was changed from Sae Mulli, which is "New Physics" in Korean, to New Physics: Sae Mulli.

The independent homepage for the journal New Physics: Sae Mulli was revamped (Fig. 1) in 2013, to allow online submission and review of papers. This also made it possible to find all information related to New Physics articles at a glance, including features such as Online First, Most Read Articles, Highlight Papers, Archives (1961 to present day), and Search functions. By including with this the editorial board member list, aims and scope, and a short description of the journal's history through which the reader can see information about New Physics: Sae Mulli, we are raising the prominence of the journal's excellent image.

A list of articles accepted for publication can be seen on the Online First section of the new homepage, and on the Most Read Articles section appears a list of the 15 most-read articles from among articles published within the past two years.

In addition, in the Highlight Papers section is listed all the top-quality research articles selected each month. In particular, in the case of cover articles, a representative picture is published every month on the journal's cover (Fig. 2), and a letter is sent notifying the author that the article will be published as a cover article.

In the Archives section, all articles from 1961 to the present are available. Here, the reader can find many articles written by scientists from earlier generations, and at the same time, can gain an understanding of the passion and hard work of these earlier generations of authors and their dedication to New Physics: Sae Mulli.

In addition, New Physics: Sae Mulli has joined CrossCheck, CrossRef, and CrossMark and has become an open-access journal. The words 'open access' appear at the bottom of the first page of every article printed, officially noting that the article is open access. Recently, the journal has revised its ethics rules, and its article submission and review regulations. In ad- dition, the English abbreviated name of New Physics: Sae Mulli was selected (New Phys.: Sae Mulli), an eISSN (2289-0041) was obtained, an Errata section was introduced, and an author checklist was established.

Along with this, the Aims \& Scope of New Physics: Sae Mulli has been revised and improved. In the spring of 2014, the Aims \& Scope was amended to include the following: "1. Review of Current Physics Topics, 2. Applied Physics, 3. Other Areas of Physics". The results these changes appear on the cover page of New Physics: Sae Mulli's, clearly improved as shown in Fig. 2.

There are currently eleven foreign editors participating in the editorial board of New Physics. They are Charles M. Falco (USA), Marco Grioni (Switzerland), Karsten Horn (Germany), Hideomi Koinuma (Japan), Antonello Tebano (Italy), Alexey Yamilov (USA), Sung Seok Ambrose Seo (USA), Masayuki Igashira (Japan), Valery Shvetshov (Russia), Nguyen Van Do (Vietnam), and Haladhara Naik (India).

Additionally, there is a native speaking-editor (E. J. Button, USA) whose job is to proofread and edit the English articles. Through such efforts, New Physics: Sae Mulli is continuing to advance with new developments. We look forward to the on-

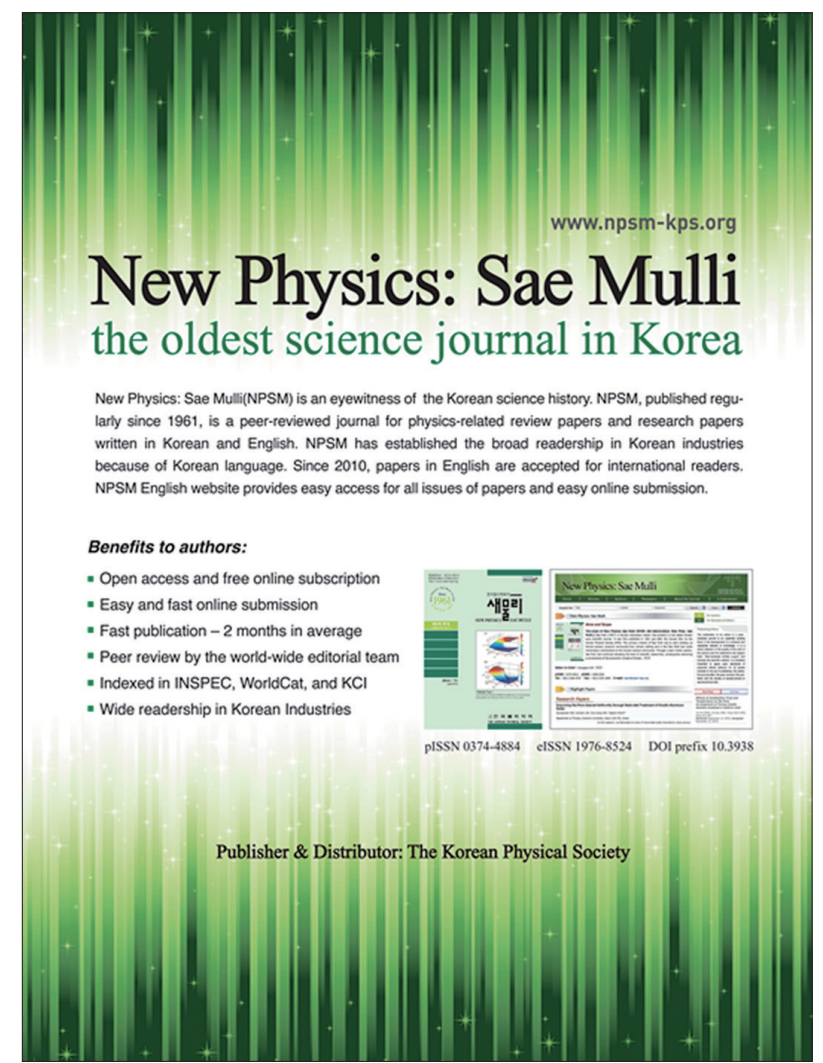

Fig. 3. Promotional material for New Physics: Sae Mulli posted in the Association of Asia Pacific Physical Societies Bulletin. 
science editing /

going elevation in status of New Physics: Sae Mulli.

The journal New Physics: Sae Mulli publishes about fifteen articles on the last day of the month. Review papers make up $5 \%$; condensed matter physics and applied physics papers make up 37\%; physics education, 16\%; atomic physics, molecular physics and optical science, 14\%; general theory, mathematics, and statistical physics, $12 \%$; particle and nuclear physics, 9\%; fluid mechanics, geophysics and astrophysics, $4 \%$; and plasma and chaos theory account for $3 \%$ of the papers published in the journal.

Looking at the types of articles published the most, articles on condensed matter and applied physics were the most frequently published, followed by physics education, atomic physics, particle physics, and optical science. While publishing a fixed number of articles in each monthly issue, the New Physics: Sae Mulli editorial staff strives to maintain a balance in the number of articles from each field.

In addition, we are working hard to promote the journal. Every year, prizes are awarded, including an opening prize, article prize (reflecting its impact factor), and a participation prize (the author who has published the most articles, for members aged 35 or under). In addition, a promotional pamphlet is made and distributed to members at the general academic conference convened every year. Through efforts such as the publishing of advertisements (Fig. 3) in the Association of Asia Pacific Physical Societies bulletin for international publicity, we continue to work towards bringing New Physics: Sae Mulli to an even greater level of development.

\section{Conclusion}

Using this development as a stepping stone, we will devote ourselves with even greater effort to turning New Physics: Sae Mulli into a leading global journal in both name and reality. Looking forward, we will aim to continue developing, through activities such as working towards getting indexed in SCI(E), simultaneous publishing of outstanding articles in Korean and English, establishing thematic special issues, and publishing tutorials as well as review articles.

\section{Conflict of Interest}

No potential conflict of interest relevant to this article was reported.

\section{Acknowledgments}

With respect to the indexing of the journal New Physics: Sae Mulli in Scopus, I would like to express my eternal gratitude to the generations of editors-in-chief and editors who have worked so hard over the years. 